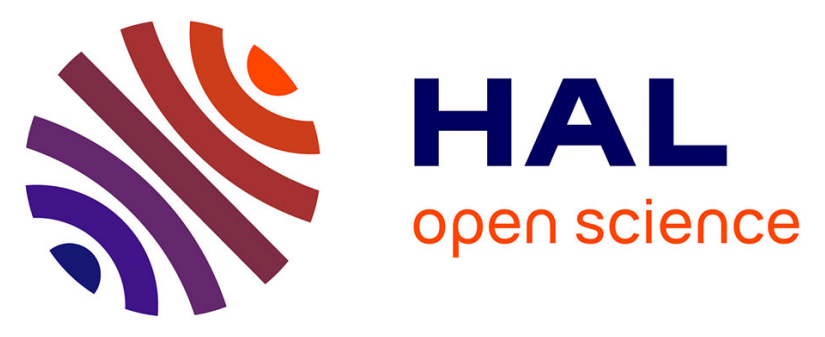

\title{
Stratégie de développement et conservation des suberaies des monts de Tlemcen (Algérie)
}

\author{
Assia Letreuch-Belarouci, Medjahdi Boumediene, Noureddine \\ Letreuch-Belarouci, Patrick Aumasson
}

\section{To cite this version:}

Assia Letreuch-Belarouci, Medjahdi Boumediene, Noureddine Letreuch-Belarouci, Patrick Aumasson. Stratégie de développement et conservation des suberaies des monts de Tlemcen (Algérie). Revue forestière française, 2010, 62 (1), pp.25-42. 10.4267/2042/32972 . hal-03449629

\section{HAL Id: hal-03449629 \\ https://hal.science/hal-03449629}

Submitted on 25 Nov 2021

HAL is a multi-disciplinary open access archive for the deposit and dissemination of scientific research documents, whether they are published or not. The documents may come from teaching and research institutions in France or abroad, or from public or private research centers.
L'archive ouverte pluridisciplinaire HAL, est destinée au dépôt et à la diffusion de documents scientifiques de niveau recherche, publiés ou non, émanant des établissements d'enseignement et de recherche français ou étrangers, des laboratoires publics ou privés. 


\title{
AMENAGEMENT ET GESTION
}

\section{Stratégie de développement \\ ET CONSERVATION DES SUBERAIES \\ DES MONTS DE TLEMCEN (Algérie)}

\author{
Assia Letreuch-BelarouCi - Medjahdi Boumediene \\ Noureddine Letreuch-Belarouci - Patrick Aumasson
}

Située exclusivement en Méditerranée occidentale, la forêt de Chêne-liège se caractérise par un fort taux d'endémisme. Elle présente ainsi une grande valeur patrimoniale, qui n'a d'égale que sa valeur économique comme source de liège, et aussi comme lieu de pâture. Son fonctionnement et la qualité du paysage qu'elle offre ont motivé pour une grande part son inscription comme habitat ${ }^{(1)}$ Natura 2000 par l'Union européenne. Elle est cependant menacée, dans les environs de Tlemcen (Algérie), par le développement mal maîtrisé d'un usage pastoral, et d'une forte pression due à la récolte des glands, cause de la disparition de toute régénération depuis une vingtaine d'années (Letreuch-Belarouci, 2002).

Préserver ce patrimoine naturel exceptionnel ne peut se faire sans la création d'un mode de développement durable, créateur d'activités et d'emplois pour les populations locales et respectueux du patrimoine naturel et culturel. Un tel espace protégé doit être régénéré et géré de façon durable afin de donner à ces communautés les moyens de conduire ce territoire, pour continuer à vivre dans la forêt selon leurs traditions. C'est donc un défi, pour le parc national de Tlemcen, que d'instaurer un nouveau mode de gestion qui puisse maintenir les activités traditionnelles sans compromettre la forêt à long terme.

\section{CONDITIONS STATIONNELLES}

Au sud-ouest de la ville de Tlemcen sur des grès séquaniens au niveau du Nord des monts se trouve un groupement comportant les deux intéressants massifs de Chêne-liège de Zariffet (962 ha) et de Hafir (9 872 ha). Ces massifs se situent à une altitude qui varie de 1000 à $1418 \mathrm{~m}$ (Parc national de Tlemcen, 2000). Ils se présentent sur un relief accidenté où toutes les expositions sont présentes. Les pentes dominantes sont de l'ordre de 12,5 à $25 \%$. Les grès ont donné naissance à des sols bruns forestiers de texture sablo-limoneuse, souvent évolués en sols fersiallitiques (Gaouar, 1998).

Le climat est méditerranéen, marqué par une sécheresse estivale qui se manifeste dès le mois de juin. Seulement 7,2 \% des $607 \mathrm{~mm} / \mathrm{an}$ de pluie tombent pendant la saison estivale (période 1970-2004). La moyenne des températures maximales du mois le plus chaud s'établit à $31,6{ }^{\circ} \mathrm{C}$, celle des minima du mois le plus froid à $2,8^{\circ} \mathrm{C}$. L'indice pluviométrique d'Emberger Q2 (Emberger,

(1) Il s'agit, pour Natura 2000, d'un habitat d'intérêt communautaire dit prioritaire (habitats ou espèces prioritaires : habitats ou espèces en danger de disparition sur le territoire européen des États membres et pour la conservation desquels l'Union européenne porte une responsabilité particulière). 


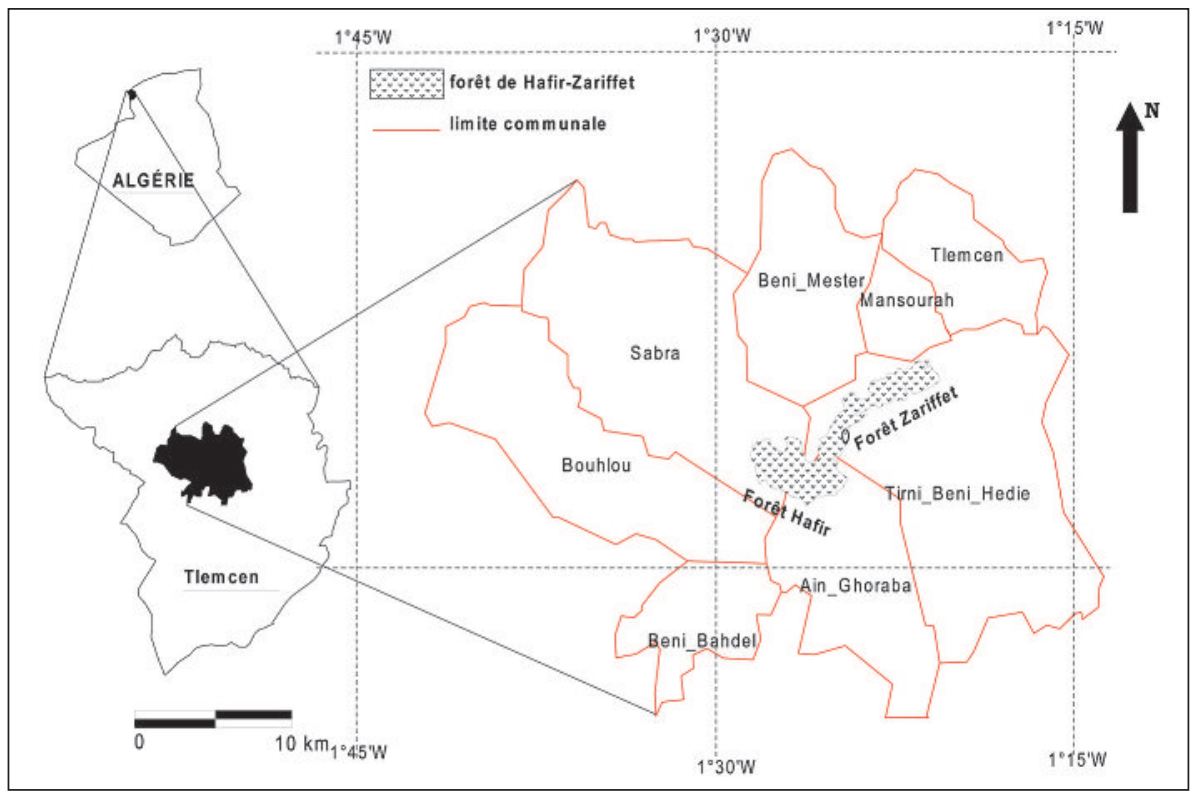

1939) est de 84,5 , ce qui permet de classer ce climat dans le subhumide inférieur, à variante fraîche. L'enneigement est aussi présent sur l'ensemble du massif au-delà d'une altitude de $1200 \mathrm{~m}$, le nombre de jours de neige varie de 7 à 25 avec une couche moyenne de 10 à $20 \mathrm{~cm}$ d'épaisseur (Benabdeli, 1996).

Du point de vue juridique, le massif de Hafir-Zariffet relève dans sa totalité du régime juridique domanial et se trouve de ce fait sous la gérance de la Direction générale des Forêts, sa soumission au régime forestier a été acquise dans la période 1868-1899. Sa gestion est assurée par le parc national ainsi que par les circonscriptions des forêts de Tlemcen, de Maghnia et de Sebdou.

\section{L'histoire de la suberaie du parc national}

La forêt de Hafir était constituée essentiellement d'une vieille futaie de Chêne-liège de 200 à 250 ans, avec quelques taillis de cette essence provenant d'exploitations de guerre ; on ne trouvait que de rares traces de régénération naturelle (Boudy, 1955). Ces chênes ont subi dans le passé, en plus du vieillissement, toutes sortes de dévastations liées en particulier aux incendies, aux coupes sévères et au surpâturage. Une bonne partie a, de ce fait, irréversiblement disparu (Bouhraoua, 2003). Très éclaircis, ils renfermaient déjà dans les années 1950 une densité moyenne qui variait de 70 sujets à l'hectare, dans beaucoup d'endroits incendiés et dégradés, à plus de 300 dans les parties où l'ancienne futaie était conservée. Les incendies ont été les principaux responsables de la régression du Chêne-liège dans cette forêt. Ainsi, l'incendie de 1892 a-t-il suffi à déclencher le processus de dégradation, où une surface de 1203 ha a été ravagée, soit le quart du peuplement (COIT, 1969). Quelques étendues ont été reconstituées très partiellement par des plantations artificielles d'une part et par une reprise végétative d'autre part. En 1920 et 1956, d'autres incendies ont moins gravement compromis l'état des peuplements. En 1994, plus de 640 ha ont été détruits (CFWT, 1995). En 2001, 2004 et 2006, de nouveaux incendies ont compromis toutes les régénérations bienvenantes. 
D'autres formes de dégradation ont été signalées, il s'agit surtout du pacage incessant des troupeaux, des coupes illicites, des vols de liège pour la confection de ruches, des défrichements, etc. Les travaux sylvicoles et les repeuplements, qui visaient à améliorer la vigueur des arbres ou à les rajeunir, ont été quasiment absents. Quelques opérations sommaires d'assainissement ont toutefois eu lieu en 1978 sur 80 ha suite aux chablis qui ont été provoqués par d'abondantes chutes de neige. La régénération naturelle par semis est depuis longtemps rare et irrégulière dans l'ensemble du massif, même si elle peut être satisfaisante par endroits (Thintoin, 1948). En 1952, des essais de régénération par semis sur banquettes ont été entrepris sur une surface de 30 hectares. Boudy (1955) signalait, qu'" on s'y prend trop tard ; il y a 50 ans que l'on aurait dû commencer et tenter en même temps le recépage des arbres les moins âgés. Malheureusement cette forêt, qui autrefois produisait le meilleur liège d'Algérie, est-elle nettement sur son déclin ».

La forêt de Zariffet quant à elle était constituée au début du siècle dernier de peuplements de Chêne-liège de 80 à 90 ans, très pauvres et mal venants suite à un incendie recensé en 1892 qui a parcouru environ 450 ha $(A E F C O, 1912)$. Ils se trouvaient déjà dans les années 1950, dans un état de dégradation assez avancé du fait de la croissance très ralentie des arbres, en relation avec la trop forte densité des tiges non éclaircies et le démasclage trop haut des arbres (Boudy, 1950). Les grands incendies de 1966, 1983, 1994, 2004 ont parcouru presque la totalité de la forêt et l'ont transformée en un paysage dégradé. Peu de travaux sylvicoles ont été appliqués. Quelques travaux d'ouvertures de tranchées pare-feu et d'aménagement de pistes ou d'assainissement ont eu lieu à partir de 1977. La régénération naturelle par semis, faible partout, a été cependant meilleure sur les versants exposés au nord.

Depuis cette époque, et plus récemment, l'étude de la typologie du massif de Hafir-Zariffet (Benmostefa, 2004 ; Derbal, 2006 ; Ghalem, 2006 ; Letreuch, 2009) témoigne de l'existence d'une suberaie dégradée par l'action anthropique, d'une surexploitation passée du liège et de l'absence de travaux sylvicoles proprement dits. Les auteurs précités démontrent que, contrairement à ce qui était constaté dans le passé, les classes d'âges inférieures à 50 ans sont bien représentées du fait du passage successif des feux. La suberaie est dans son ensemble très jeune, elle résulte d'une régénération naturelle (rejets de souches) provoquée par les incendies qu'a connus la région pendant ces dernières années. La présence de perches et de très petits bois reflète bien cette situation. Par contre les semis sont de densité très faible : ils n'assurent pas pour le moment le renouvellement des peuplements.

Le ramassage systématique des glands qui sont doux et comestibles, en général avant même leur maturité, constitue dans beaucoup de régions subéricoles un handicap majeur et une contrainte de taille pour la régénération naturelle par semis (Bendaanoun, 1998). Le surpâturage qui se traduit par des charges pastorales excessives et incontrôlées a aussi un effet néfaste sur les suberaies et entrave toute régénération et ce par broutage répété, piétinement et tassement du sol qui devient mal aéré, compact avec une infiltration d'eau de pluie très limitée.

\section{Dynamique du massif de Hafir-Zariffet}

La série méso-méditerranéenne du Chêne-liège est fortement représentée à Hafir, elle est visible à travers les groupements suivants :

- groupement arbustif de Chêne-liège, Chêne vert et Chêne zéen ;

- groupement arborescent de Chêne-liège, Chêne vert avec un faciès à Ciste ;

- groupement arborescent de Chêne-liège, Chêne vert et Chêne zéen ;

- groupement arborescent de Chêne-liège et Chêne vert à faciès à Lentisque. 
À ce propos, les relevés phytosociologiques effectués par Dahmani (1984) et Zeraïa (1981) au niveau du massif de Hafir ont permis de le rattacher à l'association de type Cytiso triflori-Quercetum suberis $\mathrm{Br} . \mathrm{Bl}$ et à la sous-association Quercetosum canariensis. L'allure de la végétation la plus évoluée est celle d'un matorral arboré élevé et dense. Cependant, tous les auteurs qui décrivent l'association du Chêne-liège s'accordent sur le point suivant : " cette association présente dans les différentes suberaies résulte d'une perturbation anthropique très profonde, elle est donc loin d'être climacique ». Ainsi Natividade (1956) nous décrit la nature de ces perturbations tels que la faible densité des peuplements, les élagages, les labours, le dessouchement...

Les phytosociologues sont aussi divisés en ce qui concerne la liaison entre l'association du Chêne-liège et du Chêne vert. Pour Braun-Blanquet dans l'étude de la végétation du massif des Maures, l'association Quercetum suberis est subordonnée à Quercus ilicis galloprovincialis, et le Chêne-liège $y$ est en mélange avec le Chêne vert et forme une association typique. Pour Sauvage (1961) et Emberger (1936), le Chêne-liège et le Chêne vert sont deux espèces de climax, ayant leur association spécifique, ces deux espèces peuvent coexister dans des conditions écologiques spéciales qui font qu'elles se tolèrent.

De plus, la strate arbustive de la suberaie de Hafir se compose essentiellement de Quercus suber L., Quercus rotundifolia L., Quercus faginea ssp tlemceniensis Lamk et Juniperus oxycedrus L. Par contre, le sous-bois est peu développé, il est composé principalement d'espèces caractéristiques du groupement de la chênaie mixte avec : Cistus salvifolius L., Cistus monspeliensis L., Erica arborea L., Lavandula stoechas L., Arbutus unedo L., Lonicera implexa Aiton, Viburnum tinus L., Genista tricuspidata Desf., Rosa canina L., Ruscus aculeatus L., Asparagus acutifolius L., Ampelodesmos mauritanicus (Poir.) T. Durand et Schinz, Stipa tenacissima L., Rosmarinus tournefortii (de Noé ex. Jord \& Fourr.) Jahand \& Maire., Chamaerops humilis L., Calycotome villosa (Poir.) Link., Cytisus triflorus L'Her. et Pteridium aquilinum (L.) Kuhn.

Le groupement de Chêne zéen est présent sur les vallons frais, en raison de son amplitude écologique large. Quant au groupement de Chêne vert, il domine aux expositions sud de Hafir, présentant des conditions édapho-climatiques spécifiques marquées par un sol superficiel, un affleurement rocheux très apparent et une semi-aridité de son bioclimat. C'est une essence sciaphile qui craint l'hydromorphie même temporaire (Messaoudene, 1996). Les espèces qui accompagnent le Chêne zéen sont dans ce cas Pistacia lentiscus L., Juniperus oxycedrus ssp rufescens L., Olea europaea L., Ceratonia siliqua L. et Chamaerops humilis ssp argentea L.

D’une manière générale, la forêt de Hafir à l'instar de toutes les suberaies de l'Ouest forme une entité assez spéciale qui doit à tout prix être aménagée. Ainsi le Chêne zéen à la faveur d'un éclaircissement plus important et de trouées se régénère assez bien et a tendance à envahir le Chêne-liège qui se trouve évincé par le couvert épais du Chêne zéen. En conséquence, de nombreux chênes-lièges se trouvent morts, gisants au sol dans un sous-bois dense composé de Bruyère, Laurier tin et diss.

À Zariffet, les chênes-lièges émergent, assez espacés les uns des autres. Ils sont en concurrence avec l'Oléo-lentisque sur les sols argileux, le Chêne vert sur les sols calcaires recouverts d'une couche décalcifiée assez épaisse de 1 à $3 \mathrm{~m}$ (Maire, 1926) et le Chêne zéen dans les parties humides d'altitude.

En fait, le Chêne-liège est une espèce qui, même dans son aire de répartition, est confrontée à des concurrents contre lesquels elle se maintient difficilement. En aucun cas, cette espèce n'est conquérante. Un de ses atouts est sa résistance aux incendies meilleure que certains de ses concurrents. 
L'association Quercetum suberis se présente normalement sous la forme d'une futaie à deux strates :

- la strate arborescente est constituée d'arbres assez espacés dans le sub-humide ;

- la strate arbustive est plus dense que la strate arborescente.

Le groupement de Chêne-liège situé au nord-ouest se présente en premier lieu sous la prédominance d'une suberaie à Erica arborea L. dans les clairières et versants ensoleillés (tempérament héliophile), composée de Quercus suber L., Erica arborea L., Genista tricuspidata L., Lavandula stoechas L., Phillyrea angustifolia L., Arbutus unedo L., Cytisus triflorus L'Her., Cistus salvifolius L., Ampelodesmos mauritanicus (Poir.) T. Durand et Schinz, Asparagus acutifolius L. Là où l'hygrométrie est plus élevée, la présence du Chêne vert est plus marquée dans la suberaie avec quelques sujets de Chêne zéen mêlés à Quercus coccifera L., Genista tricuspidata Desf., Lonicera implexa Aiton, Dactylis glomerata L. et Carex halleriana Asso.

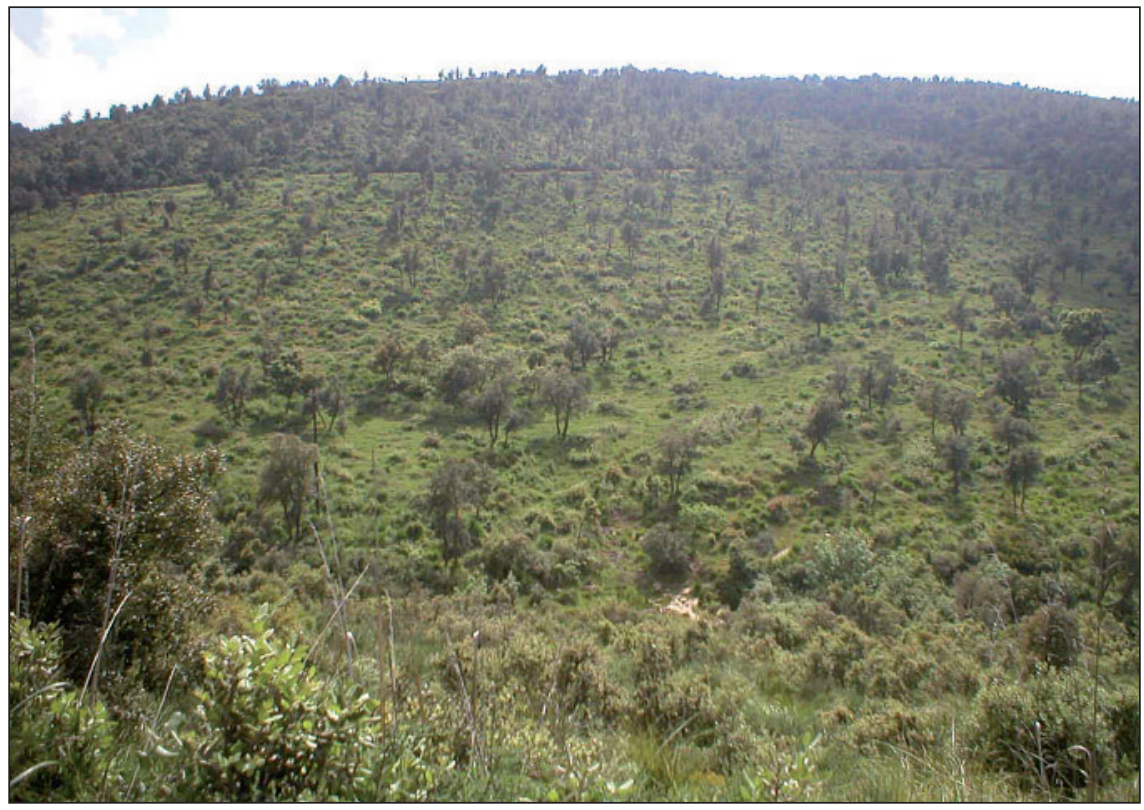

Photo 1 Aspect spectaculaire de la forte dégradation de la suberaie de Zariffet Photo Letreuch (2005)

\section{JUSTIFICATIONS POUR UNE DÉMARCHE DE RÉHABILITATION}

\section{Les menaces actuelles}

Les agents de dégradation directe des suberaies du parc national sont bien connus :

- l'incendie répété, souvent volontaire, lié aux pratiques des pasteurs pour favoriser la repousse de l'herbe après les orages de fin d'été ;

- la prédation par le troupeau, prélevant les jeunes rejets tendres et la totalité des glands qui constituent un excellent fourrage ;

- le non-rajeunissement des vieilles souches et la déficience d'une régénération naturelle par semis ;

- l'absence de plan de gestion et d'aménagement. 
Les démasclages inappropriés du liège, la pauvreté et le manque d'opportunités économiques pour les populations riveraines, l'absence de financement intégrant les questions environnementales et sociales dans les pratiques de gestion et d'aménagement et les fluctuations du marché du liège, sont à l'origine du déclin de la suberaie méditerranéenne.

\section{Services environnementaux et socio-économiques}

Depuis plus d'un siècle, la combinaison de politiques inadaptées, de mauvaises pratiques de gestion des suberaies et les évolutions du marché du liège ont entraîné la dégradation et la perte des suberaies. Afin d'améliorer les conditions nécessaires à l'équilibre écologique, social et économique de ces écosystèmes, une gestion durable et équilibrée pour la sauvegarde du Chêneliège doit voir le jour. II ne reste aujourd'hui que le quart des 3 millions d'hectares des suberaies auparavant présentes au Maghreb (WWF, 2004).

Les suberaies de Tlemcen constituent, en outre, un exemple remarquable et rare d'interaction entre l'homme et la nature. Si elles sont bien gérées, elles permettraient d'assurer des services environnementaux, comme la protection et l'entretien des sols et des eaux ; la recharge de la nappe phréatique et le contrôle des eaux de ruissellement ; la protection contre les incendies ; la disponibilité d'habitats pour la biodiversité, par exemple des zones de reproduction et de nidification pour les oiseaux migrateurs (WWF, 2004).

Elles sont aussi à la base d'un système économique varié et rentable reposant sur :

- l'extraction et traitement du liège pour la fabrication des bouchons et l'élaboration de matériaux de construction ;

- les produits de l'élevage et de l'agriculture ;

- la récolte du bois, des champignons, des glands, du miel, la cueillette des plantes médicinales et aromatiques, etc. ;

- le tourisme culturel et écologique.

Au-delà d'assurer un revenu pour une centaine de milliers de personnes dans la région, les suberaies abritent une biodiversité extrêmement riche, constituée notamment d'espèces menacées.

Enfin, comme toutes les forêts cultivées, elles participent à la lutte contre l'effet de serre.

\section{Production de liège}

La production de liège dans la région dépend principalement de la forêt de Hafir dont l'étendue est la plus importante. Le liège de reproduction (ou liège femelle formé lorsqu'on dépouille l'arbre de son premier liège mâle) constitue le véritable liège de commerce, depuis longtemps considéré comme un liège de qualité supérieure, et il représentait les deux tiers de la production locale qui, selon Boudy (1955), avait atteint 20 ooo quintaux entre 1939 et 1951 alors que le liège mâle représentait un tiers seulement du total (6 300 quintaux).

L'exploitation du liège a été réalisée avec une rotation de 10 à 12 ans, par jardinage ou par coupon, et le canton (figures 2 et 3, p. 31), quelle que soit sa superficie, était considéré comme une unité de division car un seul canton était concerné à chaque campagne de récolte. La surface actuellement productive à Hafir est d'environ 1069 ha, ce qui représente $31 \%$ de la superficie initiale (Bouhraoua, 2003). La production de liège femelle à Zariffet entre 1939 et 1951 (4 700 quintaux) représentait le quart de ce qui a été récolté à l'échelle de la région (Boudy, 1955). En 1981, 2268 quintaux de liège de reproduction seulement ont été levés à cause d'un grand incendie qui a éprouvé cette forêt (AEFCO, 1995). La superficie encore productive à Zariffet 


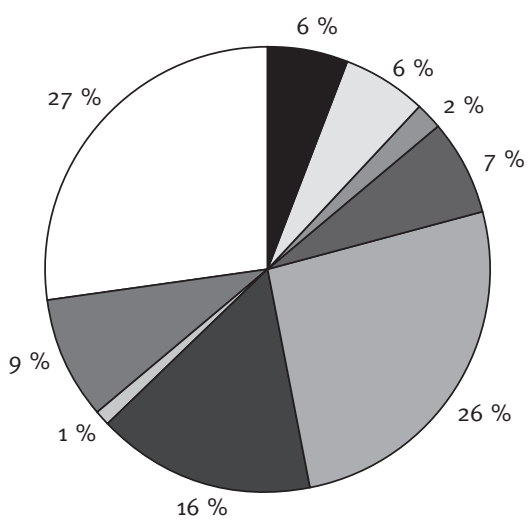

canton Maison forestière

canton Hafir

canton Bled Aïni

canton Tibrouine

canton Oued Tlet

canton Tijdit

canton Krean

canton El Kroum

canton Tatsa

est insignifiante puisqu'elle est de l'ordre de 18 ha. En 2008, on a enregistré pour l'ensemble de la région une production de l'ordre de 205 quintaux (Direction générale des Forêts, 2008).

La chute sensible de la production en liège s'explique d'une part par la régression de la surface productive et d'autre part par la vigueur des arbres en production qui se détériore avec le temps du fait de l'action anthropique. D'autre part, on peut affirmer que les peuplements affectés par le feu recèlent une densité importante de petits bois (diamètre entre 8 et $22 \mathrm{~cm}$ ), qui sont en phase de récupération après les ravages causés par les incendies récents (2004 et 2006). Leurs houppiers se reconstituent lentement et ne produisent que très peu de glands et de liège pour le moment.

L'histoire très récente des incendies (2004) mais aussi bien lointaine de ce massif montre une dégradation du milieu et une régénération par semis de plus en plus faible. L'état actuel des peuplements est principalement une conséquence des incendies répétés et de la surcharge de l'élevage traditionnel qui y est pratiqué. Ces pressions ont eu pour résultat la formation de

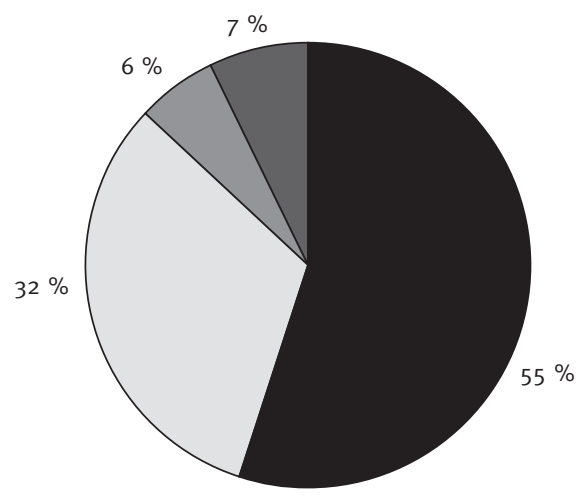

canton Zariffet

canton Ain Mardjen

canton Fernana

canton Guendouza 
peuplements irréguliers fragmentés, où une régénération par rejet de souche commence à évoluer (Medjahdi et al., 2008).

Des mesures de conservation telles que les réserves intégrales dans le but d'une conservation in situ des ressources génétiques de Quercus suber doivent être plus actives, et avoir comme objectifs de gestion, le renouvellement de l'espèce, l'augmentation de la diversité génétique et le contrôle de l'équilibre entre les différentes espèces.

\section{CONSTAT ACTUEL}

En absence d'interventions sylvicoles, la suberaie est une forêt menacée de disparition car elle a des relations très étroites avec l'homme. Si elle est abandonnée, les essences secondaires et les ligneux du sous-bois ferment le peuplement et éliminent peu à peu le Chêne-liège. Le maintien de la suberaie dépend en grande mesure de la possibilité de la continuation ou de la reprise de la subériculture, donc de l'action de l'homme (Chevallier, 2002).

Les suberaies de Tlemcen ne sont ni aménagées ni correctement gérées, induisant une récolte de liège qui diminue chaque année et atteint un niveau très faible par rapport au potentiel de production qui, bien que très marqué par les incendies, reste important. La remise en production rationnelle du patrimoine subéricole qui recèle une richesse potentielle importante nécessite d'après Yessad (2000) :

- un important investissement du réseau de desserte ;

- une protection efficace contre les incendies et le surpâturage ;

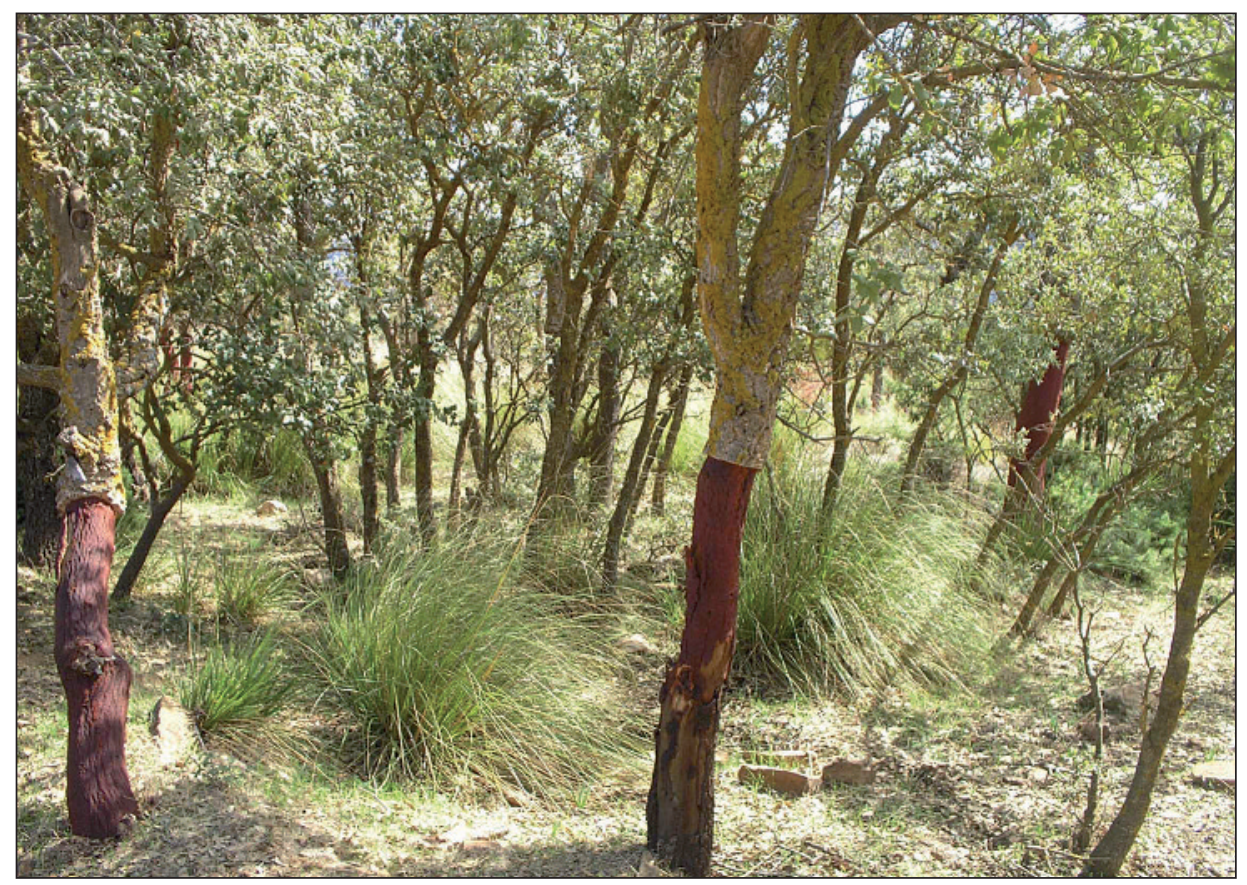

Photo 2 Suberaie de Hafir dégradée non aménagée. Remarquer la forte densité des perchis non éclaircis 
- la récupération des zones de production ;

- la modification des règles de récoltes et de culture.

Restaurer la suberaie naturelle du parc partout n'est ni possible, ni judicieux. Nous avons besoin d'un plan d'action différencié répondant aux besoins d'un pays montagneux densément habité. L'alternative n'est pas de protéger ou d'exploiter, il s'agit de protéger et d'exploiter. L'objectif est de conserver partout une forêt aussi naturelle que possible, capable de remplir simultanément plusieurs fonctions : une forêt multifonctionnelle.

La stratégie à appliquer dans le parc se base sur trois principes complémentaires :

- une sylviculture proche de la nature sur l'ensemble de la surface forestière ;

- des interventions ciblées pour favoriser la diversité biologique et structurelle, par exemple dans des réserves de forêts spéciales ;

- des réserves de forêts naturelles (réserves intégrales) soustraites à toute intervention.

En outre, l'espace forestier de l'Algérie, qui était vivrier pour une grande partie de la population, est affecté aujourd'hui par un processus de déstabilisation et de dégradation. L'occupation coloniale, la guerre de libération nationale et les retards accumulés pour la préservation et le développement réel de ce secteur ont fait que ce patrimoine tend progressivement vers un rétrécissement pouvant compromettre, à moyen ou long terme, les équilibres écologiques et socioéconomiques (Anonyme, 1988).

Le manque d'interventions sylvicoles en vue d'un rajeunissement et l'absence d'un aménagement propre aux suberaies justifient la régression de la production nationale en liège. En outre, les années 1994 et 1995 sont les plus désastreuses pour les formations de Chêne-liège tant au plan du rendement en liège qu'au plan de leur avenir, cette situation découle de l'insécurité qu'a connue l'Algérie sur une décennie.

Comme perspective, selon la Direction générale des Forêts, la production devra augmenter dans des proportions sensibles dans les années à venir grâce à l'amélioration des conditions d'accès à certains massifs. À plus long terme en 2040 , le programme de rénovation de la suberaie, déjà engagé à travers l'étude prospective du secteur forestier en Afrique (FOSA) établie par la FAO, devrait permettre d'améliorer les performances et donc de faire face à la consommation locale et d'augmenter la part des exportations afin d'améliorer la balance "bois et produits non ligneux" (Goussanem, 2000).

\section{STRATÉGIE DE DÉVELOPPEMENT}

Les politiques forestières menées depuis ces trois dernières décennies n'ont pas eu pour conséquence la préservation du potentiel forestier existant et cela est dû à deux raisons essentielles d'ordre :

- technique, l'absence d'aménagement des forêts ;

- anthropique, l'absence de prise en compte du facteur humain.

En effet, les premiers programmes d'études d'aménagement n'ont été mis en place qu'à partir de 1982 et leur application demeure toujours très limitée, voire nulle, d'où l'absence d'une gestion technique stable et durable. Quant au facteur humain, il n'a été que très rarement pris en considération dans les différentes stratégies de développement forestier, et il demeure la cause principale des échecs enregistrés (Noual, 1996). Il faut souligner également que les plus grands programmes de travaux forestiers ont été attribués à l'administration des forêts qui n'a jamais mis en place suffisamment de moyens humains et matériels pour finaliser ses projets. 
Ainsi, toutes les politiques se fondent sur l'attribution à l'administration des forêts de la totalité des initiatives et du contrôle des activités forestières, tant sectorielles que centralisées.

Noual (1996) précise à ce sujet : " devant le peu de résultats obtenus par les politiques forestières antérieures et l'incapacité qu'a eue l'administration de gérer certaines situations, il s'impose alors de rechercher de nouvelles stratégies, intégrant au mieux les aspirations des populations riveraines, dans le cadre d'un nouveau système permettant une plus large participation. Le rôle, à cet égard, de l'administration doit donc largement être repensé et la gestion instituée par les politiques forestières, moins centralisée qu'elle n'apparaît aujourd'hui. Ces nouvelles stratégies doivent nécessairement privilégier des modèles sylvicoles consistant à améliorer la gestion des peuplements existants, en rendant les utilisateurs riverains plus responsables de leurs pratiques ».

Pour répondre à l'ensemble de ces objectifs, il est proposé de créer un réseau de réserves sur une partie du territoire du parc. C'est en premier lieu un territoire reconnu d'intérêt majeur, que l'on souhaite préserver et gérer de façon durable pour les hommes d'aujourd'hui et pour les générations futures. Pour cela, il est impératif de délimiter sur le terrain différents types de zones ayant pour vocation la protection de la nature, la recherche scientifique, le soutien des cultures locales, ou encore le développement d'activités économiques locales. On définit pour chacune d'elles des règles de gestion.

Il est d'abord nécessaire de définir un plan d'actions reposant, d'une part, sur des interventions de type sylvicole en vue de la mise en place d'un plan d'aménagement durable et, d'autre part, sur l'introduction de systèmes agroforestiers dans les zones limitrophes aux villages, à fort et moyen impact humain. Ces suberaies doivent jouer un rôle multifonctionnel satisfaisant à la fois les intérêts sylvicoles, écologiques et socioéconomiques. C'est pour cela que nous proposons trois types de vocation : la production soutenue de liège dans des réserves forestières particulières ; la protection du Chêne-liège par la création de réserves forestières naturelles ; l'agroforesterie.

Pour augmenter et conserver la diversité spécifique sur l'ensemble du territoire du parc, il est nécessaire d'appliquer une gestion orientée vers la restauration d'écosystèmes complets sur des espaces spécialisés et limités, ainsi que des dispositions plus légères mais généralisées à l'ensemble des forêts.

Les choix du parc national se portent sur les méthodes de sylviculture qui assurent au mieux :

- la protection physique des sols et l'amélioration de leur capacité de production ;

- la qualité et la stabilité des paysages ;

- la diversité des écosystèmes, à l'échelle du massif, de la forêt et de la station ;

- la conservation des ressources génétiques des essences autochtones.

La régénération sera naturelle, lorsque les peuplements en place sont composés d'essences spontanées bien adaptées aux stations. Une orientation vers la futaie irrégulière et mélangée est souhaitable pour créer une mosaïque de peuplements favorables au développement de la diversité biologique et à la préservation des paysages (Aumasson, 1997). Le régime du taillis simple sera cependant maintenu pour satisfaire les besoins des populations locales notamment en bois de feu. On veillera cependant au renouvellement de l'ensouchement indispensable à la poursuite durable de ce traitement.

La régénération naturelle sera préférée à la régénération artificielle, car elle permettra de faire évoluer progressivement les peuplements dans une continuité dynamique de maturation, en offrant une forte capacité de résistance aux attaques parasitaires. Lorsque les essences présentes ne répondent qu'imparfaitement aux objectifs écologiques, paysagers et économiques, la régénération naturelle pourra être assistée de façon à enrichir, par des compléments adaptés, la composition des peuplements. 


\section{Propositions concrètes de renouvellement}

\section{- Forêt de Hafir}

On peut assigner à cette forêt trois vocations majeures : protection-production, agroforesterie et réserve intégrale (figure 4 , ci-dessous) :

- la réserve forestière à production soutenue de liège comprend les peuplements de Chêneliège pur ou en mélange, se trouvant en état bon ou jugé récupérable. Il s'agit des cantons Oued Tlet et Tibrouine ;

- le système d'agroforesterie comprend les terrains situés à proximité des villages. II s'agit d'une partie des cantons de la maison forestière de Hafir et Tatsa ;

- les réserves forestières naturelles concernent les cantons Tijdit, Bled Aïni, une partie de Tatsa et El Kroum.

FIGURE 4

CARTE DES DIFFÉRENTES ZONES

\section{DEVANT BÉNÉFICIER D'INTERVENTION DE DÉVELOPPEMENT}

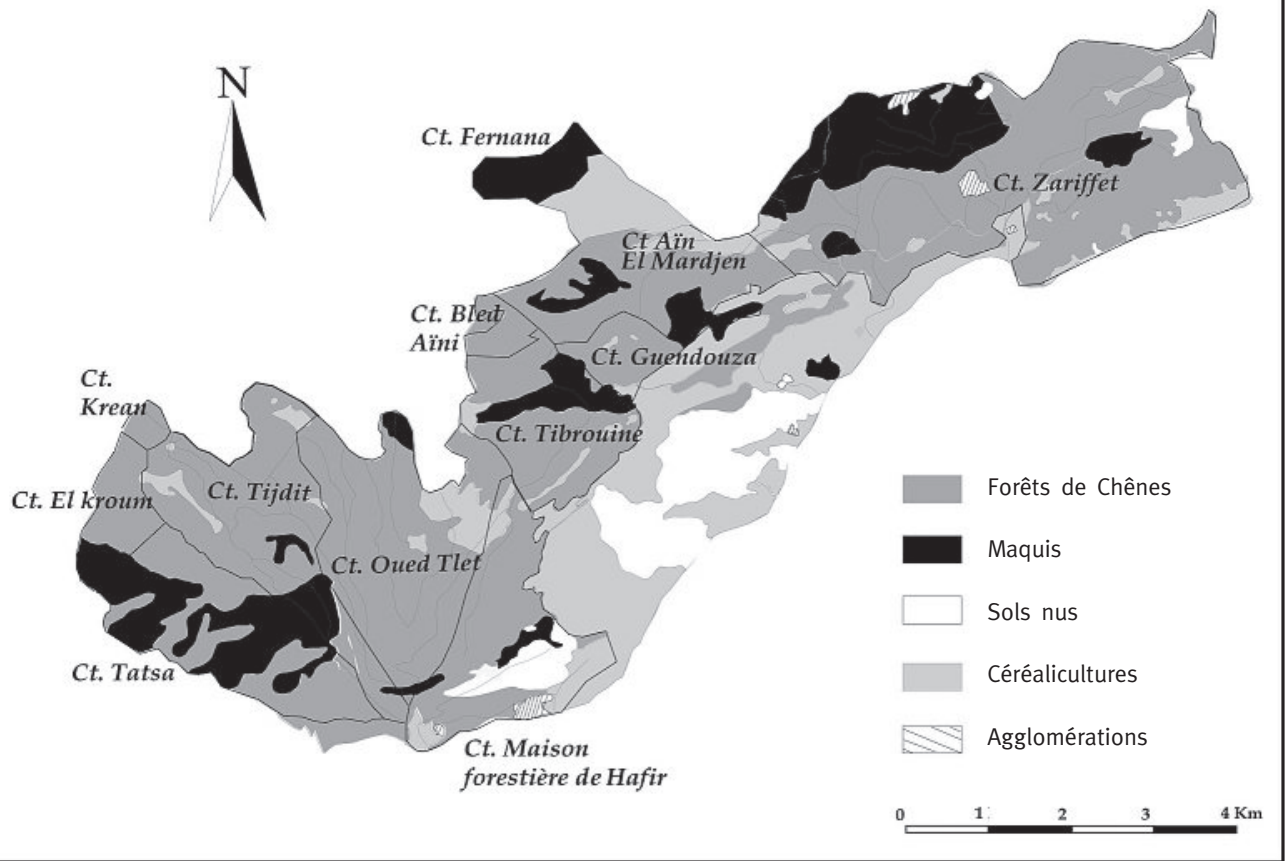

Dans les réserves forestières, un certain nombre de pratiques sylvicoles ainsi que l'exercice de la chasse restent autorisés, ce qui n'est pas le cas dans les réserves naturelles. Les réserves forestières ont un règlement beaucoup moins strict puisque notamment l'exploitation des bois y reste autorisée (Warge et Dufrene, 1996). Une de leurs fonctions consiste à servir de laboratoire pour l'enseignement et la recherche : la dendrométrie, les mesures de productivité des essences feuillues indigènes, l'étude du comportement des semis, les études pédologiques, climatologiques et faunistiques peuvent y être poursuivies.

De ce fait, elles pourraient même être appelées à jouer un rôle économique dans l'avenir. Les réserves forestières seront gérées conformément aux principes de gestion soutenue afin d'optimiser les aspects sociaux, économiques et environnementaux du pays. Au niveau de la réserve 
forestière à production soutenue de liège, les opérations à appliquer tournent autour de la mise en valeur du liège par la sylviculture. Toutefois, l'opération de démasclage ou de récolte de liège, simple en théorie, demande soin et adresse ; elle ne devra jamais être confiée qu'à des ouvriers expérimentés, car toutes les fautes commises se paient plus tard, souvent par la mort de l'arbre.

Aussi, pour ne pas reproduire les erreurs du passé, il est conseillé de ne pas dépasser, même dans des conditions particulièrement favorables, une hauteur totale de déliégeage (hausse) de $4 \mathrm{~m}$. Cette hauteur ne doit jamais être supérieure à deux fois la circonférence de l'arbre à 1,30 m au moment de la première récolte. La hausse de déliégeage dépend de l'épaisseur du liège au moment de la récolte. Si l'épaisseur du liège est inférieure à $20 \mathrm{~mm}$, il faut diminuer la hausse. Si cette épaisseur est supérieure à $27 \mathrm{~mm}$, on affectera la hausse d'un coefficient de déliégeage pour chaque classe de productivité (tableau I, ci-dessous). Dans les pays du Maghreb, la hauteur de déliégeage est de $1,8 \mathrm{~m}$ environ.

TABleau I

Coefficient de déliégeage en fonction des classes de productivité (Yessad, 2000)

\begin{tabular}{|c|c|}
\hline Classe de productivité & Coefficient de déliégeage \\
\hline Très favorable ............................. & 3 \\
\hline 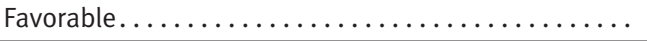 & 2,5 \\
\hline Moyenne.... & 2 \\
\hline 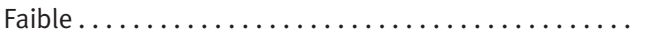 & 1,5 \\
\hline
\end{tabular}

La réserve forestière naturelle (protection intégrale) est administrée principalement aux fins d'études scientifiques et de surveillance continue de l'environnement. Elle peut servir à préserver les ressources génétiques du Chêne-liège dans les conditions les plus naturelles ou les moins modifiées qui soient. Elle représente également un des éléments structurants du paysage méditerranéen. Nous essayerons de réduire au minimum les perturbations en limitant l'accès au public et au cheptel. Ceci permettrait de suivre l'évolution des glandées (production de glands) qui est très irrégulière d'une année à l'autre. D'autre part, cette réserve jouera un rôle de verger à graines capable de fournir des glands pour des futures opérations de reboisement.

Quant au système agroforestier, il est appliqué afin de pallier le déficit fourrager, accru par les interventions en forêt, et d'intensifier les activités agricoles de la population pour augmenter son niveau de vie. La constitution de prairies permanentes à base de cultures fourragères semble intéressante. La transformation des surfaces sous-exploitées en surfaces cultivées plus intensivement (arboriculture, cultures fourragères complémentaires, maraîchage) est possible. Ainsi, les forestiers et les agriculteurs souhaitent un nouvel aménagement de l'espace, susceptible de résoudre les problèmes auxquels ils ont à faire face. Les mesures réglementaires, comme l'introduction de nouvelles cultures, doivent être élaborées de manière concertée par le forestier et l'agriculteur pour que se développent des modes de gestion agro-sylvo-pastoraux, capables de répondre à tous les besoins de la population.

\section{- Forêt de Zarifett}

Le but est ici de stimuler et dynamiser la régénération naturelle. Le traitement sylvicole envisagé est la futaie jardinée, modulée selon le zonage suivant (figure 4, p. 35) :

- zone incendiée : cette zone sinistrée (canton Zariffet) doit être clôturée en première urgence pour protéger la régénération naturelle, éventuellement assistée par l'introduction de plants d'origine locale ; 
- zone de protection ou de mise en défens. Cette zone concerne les peuplements dont l'état est devenu critique (canton Fernana et canton Ain El Mardjen), et nécessite également à court ou moyen terme la mise en place d'une clôture, physique ou écologique avec des espèces écran ;

- zone de mise en valeur. Cette zone à vocation économique concerne les peuplements dont l'état n'est pas critique et supportant encore le démasclage, le cas échéant après mise en repos et opérations sanitaires (une partie des cantons de Zariffet et Ain El Mardjen).

Une organisation pastorale dans le massif est envisageable par une articulation de l'espace en plusieurs secteurs (Yessad, 2000). Dans les secteurs à interdiction stricte, les parcelles mises en rénovation et en restauration seront strictement interdites au pâturage pendant 6 à 7 ans pour les pâturages bovins et ovins, quant aux caprins ils ne seront admis qu'après 12 ans. Une mise en défens intégrale est indispensable pendant les deux premières années. Pour les secteurs à pâturage admis, les parcelles mises en jardinage avec un complément de régénération par semis de glands seront admises à un pâturage ovin. Le bétail sera exclu des zones semées. Un pâturage bovin et ovin sera totalement admis après la sixième ou la septième année si la régénération est hors de portée de la dent du bétail. Enfin, dans les secteurs à pâturage contrôlé, le reste du peuplement sera pâturé normalement avec un contrôle de la charge. Les caprins doivent être contrôlés et admis uniquement en nombre restreint.

En outre, on doit rechercher les solutions où l'élevage peut contribuer à l'entretien de la forêt comme le parcours arboré. Il ne s'agit pas de compter sur le bétail pour débroussailler la forêt mais de développer toutes les situations où une synergie peut être trouvée entre les impératifs de production des exploitations et les impératifs de gestion de la forêt (Yessad, 2000).

D’autre part, il est préconisé de mettre en place des mesures passives contre les incendies, notamment dans les zones d'écorçage. Dans ce cas, il est recommandé de réaliser des débroussaillements par placette, au moins deux ans avant l'écorçage et de les maintenir en parfait état pendant les trois ans qui suivent la levée. En effet, les opérations préventives telles que le débroussaillement peuvent limiter les départs de feu, en faisant disparaître le principal aliment des incendies; débarrasser le sol des plantes qui s'y nourrissent au détriment des bonnes essences ; favoriser la régénération des peuplements et enfin faciliter les opérations sylvicoles (Lambert, 1860).

Il est conseillé aussi, d'après le code international des pratiques subéricoles, de compartimenter, grâce à des pare-feux, des superficies de 50 ha au maximum (CIPS, 2005).

En outre, les aménagements à apporter au niveau du massif de Hafir-Zariffet doivent permettre :

- de développer le dispositif de surveillance notamment en période à haut risque : il n'existe qu'un seul poste de vigie à Hafir au niveau du Djebel Koudiat à $1418 \mathrm{~m}$. Cela reste insuffisant pour détecter tout déclenchement de feu à Zariffet ;

- d'améliorer le réseau de pistes pour permettre l'accès rapide des intervenants ;

- d'équiper des points d'eau ;

- et d'améliorer le réseau de tranchées pare-feux (il n'existe que deux tranchées pare-feux dans chacune des deux forêts).

Le brûlage dirigé et contrôlé est également un outil de gestion de plus en plus utilisé dans les pays confrontés au problème des incendies. Il présente un faible impact écologique sur le milieu naturel et il est financièrement très économique par rapport aux travaux mécaniques.

Pendant longtemps, la stratégie contre les incendies de forêts a consisté essentiellement à améliorer la lutte contre les feux naissants par le développement de la surveillance et la rapidité 
Assia Letreuch-Belaroucl - Medjahdi Boumediene - Noureddine Letreuch-Belaroucl - Patrick Aumasson

d'intervention. Mais la nécessité d'une prévention à long terme, à la fois par la gestion et l'entretien de la forêt et par la réglementation, s'est imposée depuis quelques années.

\section{RÉGÉNÉRATION ET TRAITEMENTS SYLVICOLES}

La régénération par voie de semis est dérisoire à Hafir. Ceci est dû en grande partie à la forte présence du bétail qui tasse le sol et le rend impropre à la germination des semis. À Zariffet, la régénération par semis est plus favorable, les glands qui échappent au pâturage germent en s'entremêlant à la forte quantité de litière. Le massif forestier de Hafir-Zariffet présente une exposition dominante nord avec une pente qui varie d'un endroit à un autre. Celle-ci influe directement sur l'installation et la dissémination des glands. Les rares semis observés se localisent à Zariffet en aval des porte-graines. Par contre à Hafir, les semis sont quasiment absents, seuls les glands qui se sont trouvés amalgamés et associés aux touffes de plants à épineux ont fourni des semis.

La régénération par rejet de souche est omniprésente à Hafir et à Zariffet, cela est dû en grande partie au passage de l'incendie. Cependant, il paraît difficile de se baser uniquement sur les rejets du fait que le rajeunissement obtenu par rejets ne saurait garantir indéfiniment le renouvellement des suberaies. La capacité de l'essence à rejeter de souche diminue avec l'âge. La régénération par rejets de souches n'est qu'un palliatif, la régénération de la suberaie ne sera assurée qu'à l'aide des semis naturels ou artificiels (Belghazi et al., 2001).

Le traitement sylvicole à retenir dans l'aménagement du Chêne-liège diffère en fonction des potentialités de la forêt et des objectifs à atteindre. Il s'agit notamment des conditions stationnelles, du pouvoir de régénération et de la qualité du produit attendu par l'application de l'aménagement. Mis à part le traitement en taillis simple qui ne peut être retenu que lorsque l'on recherche le liège mâle comme produit essentiel, les traitements sylvicoles à appliquer au Chêneliège sont la futaie régulière ou la futaie jardinée. La futaie régulière est généralement la plus adaptée au Chêne-liège du fait que l'un des objectifs essentiels est la production du liège de reproduction de bonne qualité (Yessad, 2001). Ce traitement sera appliqué aux peuplements situés dans des réserves à production soutenue dont nous avons évoqué le principe précédemment. Le traitement en futaie jardinée est tout à fait préconisé dans un but de protection stricte de l'espèce.

\section{CONCLUSIONS}

Les suberaies du Parc national de Tlemcen sont aujourd'hui dégradées, abandonnées et dans un état sanitaire médiocre. La pression sociale rend rapidement inopérantes les mesures de mise en défens, alors qu'elles sont les seules capables d'assurer la survie de la forêt. Le massif de Zariffet Hafir se trouve implanté dans une zone stratégique et constitue en quelque sorte un point d'attraction qui attire toutes les communes limitrophes. Il résulte de cette situation de surexploitation des ressources une désorganisation des structures de végétation et une matorralisation qui caractérisent actuellement des parties importantes des peuplements naturels de Chêne-liège.

La remise en production de la suberaie méditerranéenne et la soustraction d'une production soutenue dans un parc national est l'objectif prioritaire des gestionnaires forestiers. La sousexploitation favorise un envahissement par des broussailles denses, génératrices d'incendie qui compromettent la régénération naturelle et dévalorisent totalement le liège sur pied (Varela, 1997). En termes d’actions sylvicoles, il s'agira, soit de réhabiliter des suberaies claires vieillies, 
soit de réinstaller de nouveaux peuplements là où la dynamique est arrivée à son terme, et où le Chêne-liège a disparu.

Arrêter la régression du patrimoine subéricole doit faire partie d'un projet économique à long terme prenant en compte l'entretien de la ressource et son adaptation au contexte économique régional (Yessad, 2000). L'instauration de réserves forestières incluant impérativement la régénération naturelle et artificielle constitue bien souvent le meilleur moyen de restauration et de réhabilitation des biotopes dégradés des écosystèmes à Quercus suber $\mathrm{L}$.

Assia LETREUCH-BELAROUCI
Enseignante
Département de Foresterie
Faculté des Sciences
UNIVERSITÉ DE TLEMCEN
BP 12 IMAMA
TLEMCEN 1300 (ALGÉRIE)
(letreuch_assia@yahoo.com)

Noureddine LETREUCH-BELAROUCI
Professeur en Sylviculture
Département de Foresterie
UNIVERSITÉ DE TLEMCEN
BP 12 IMAMA
TLEMCEN 13000 (ALGÉRIE)
(letreuchnoureddine@yahoo.fr)

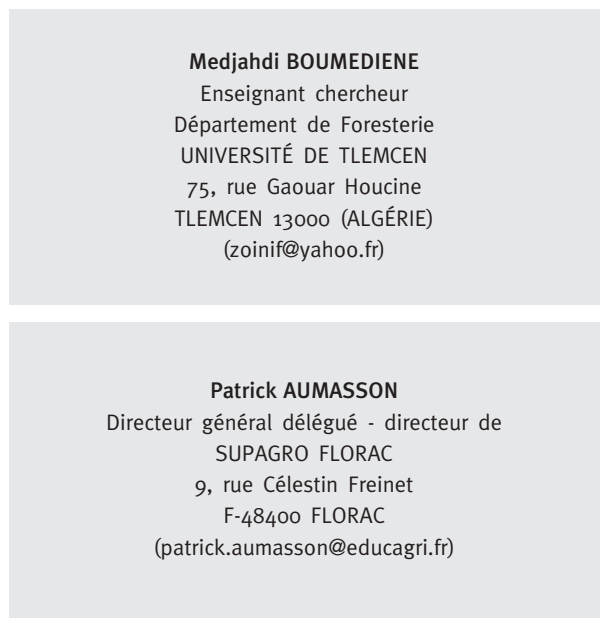

\section{BIBLIOGRAPHIE}

ADMINISTRATION DES EAUX ET FORÊTS DE LA CONSERVATION D'ORAN (AEFCO). - Description des peuplements de la forêt domaniale de Zariffet. - Cantonnement de Tlemcen, 1912. - Non paginé.

ADMINISTRATION DES EAUX ET FORÊTS DE LA CONSERVATION D'ORAN (AEFCO). - Relevés des produits constatés de la forêt domaniale de Zariffet. - Inspection de Tlemcen, 1995. - Non paginé.

Anonyme. - Espace montagne / forêt. - INESG, 1988. - $35 \mathrm{p}$.

AUMASSON (P.). - Recommandations de sylviculture pour la zone centrale du Parc national des Cévennes. - Parc national des Cévennes, France, 1997. - 57 p.

BELAGHAZI (B.), EZZAHIRI (M.), EL BOUKHARI (M.), BAKRY (M.). - Bilan actualisé des reboisements de Chêne-liège dans la forêt de la Maamora. - Séminaire du groupe IUFRO sur la sylviculture du Chêne-liège et du Cèdre. Rabat, Maroc, 2001. - pp. 161-176. 
BENABDELI (K.). - Aspects physionomico-structural et dynamique des écosystèmes forestiers face à la pression anthropozoogène dans les monts de Tlemcen et les monts de Daya (Algérie septentrionale occidentale). - Université Sidi Bel Abbes, 1996. - 356 p. (Thèse Doc. ès Science).

BENDAANOUN (M.). - Contribution à l'étude des facteurs écologiques, l'impact de la dégradation et des aménagements sur la régénération des suberaies du Rif, du Moyen-Atlas oriental et de la Mamora (Maroc septentrional). - Annales de l'INRGREF, numéro spécial, 1998, pp. 176-197.

BENMOSTEFA (O.). - Évaluation des ressources forestières de la forêt de Zariffet et proposition d'une clé de détermination des types de peuplements. - Université de Tlemcen, 2004. - 104 p. (Mémoire ingénieur forestier).

BOUDY (P.). - Économie forestière nord-africaine. Tome 2 : Monographies et traitements des essences forestières, Fasc. I et II. - Paris : Édition Larose, 1950. - 878 p.

BOUDY (P.). - Économie forestière nord-africaine. Tome 4 : Description forestière de l'Algérie et de la Tunisie. - Paris : Édition Larose, 1955. - 483 p.

BOUHRAOUA (R.-T.). - Situation sanitaire de quelques forêts de Chêne-liège de l'Ouest algérien : étude particulière des problèmes posés par les insectes. - Université de Tlemcen, 2003. - 290 p. (Thèse Doc. ès Science).

BRAUN-BLANQUET (J.). - La Végétation de l'étage alpin des Pyrénées orientales comparée à celle des Alpes. - Actas del Primer Congresso Internacional de Pireneistas, San Sebastien 5-16, 1950. - Envir Temp-Eu.

CONSERVATION DES FORÊTS DE LA WILAYA DE TLEMCEN (CFWT). - Fascicule de gestion de la forêt domaniale de Hafir, canton Oued Fernane, 1995. - 4 p.

CONSERVATION DES FORÊTS DE LA WILAYA D'ORAN (COIT). - Fascicule de gestion de la forêt domaniale de Hafir, circonscription de Tlemcen, 1969. - non paginé.

CHEVALLIER (H.). - Suberaie et biodiversité : enjeux et gestion. - VIVEXPO 2002, Biodiversité et Paysage. - France, 2002. - 8 p.

CODE INTERNATIONAL DES PRATIQUES SUBÉRICOLES (CIPS). - Fonds européen de développement régional initiative communautaire Interreg III. - France, 2005. - $12 \mathrm{p}$.

DAHMANI (M.). - Contribution à l'étude des groupements à Chêne vert (Quercus rotundifolia Lamk) des monts de Tlemcen (ouest algérien). Approche phytocoenologique et phytosociologique. - Alger : USTHB, 1984. - 238 p. (Thèse $3^{\mathrm{e}}$ cycle).

DERBAL (S.). - Typologies et éléments de gestion d'un peuplement feuillus (Chêne-liège) à Hafir (Wilaya de Tlemcen). - Université de Tlemcen, 2006. - 113 p. (Mémoire ingénieur forestier).

DIRECTION GÉNÉRALE DES FORÊTS (DGF). - Volume de liège réalisé par Wilaya arrêté au 20/01/2008. Alger, 2008. - 1 p.

EMBERGER (L.). - Aperçu général sur la végétation du Maroc. Commentaire de la carte phytogéographique du Maroc 1/1 500 000. - Veröffentlichung des Geobotanischen Institutes der Eidgenössischen Technischen Hochschule Stiftung Rübel Zürich, n 14, 1939, pp. 40-157.

EMBERGER (L.). - Présentation de la carte phytogéographique du Maroc au 1/1 500 ooo. - Compte rendus des Séances mensuelles de la Société des Sciences naturelles du Maroc, nº 4, 1936, pp. 28-29.

GHALEM (A.). - Étude typologique, stratégie de réhabilitation et réaction du milieu après incendie : cas des suberaies de Tlemcen. - Université de Tlemcen, 2006. - 94 p. (Mémoire ingénieur forestier).

GAOUAR (A.). - Hypothèse et réflexion sur la dégradation des écosystèmes forestiers dans la région de Tlemcen. - Forêt méditerranéenne, tome 2, 1980, pp. 131-146.

GOUSSANEM (M.). - L'Étude prospective du secteur forestier en Afrique (FOSA). Algérie. - Rome : FAO, 2000. - 60 p. [en ligne ftp://ftp.fao.org/docrep/fao/003/X6771F/X6771Foo.pdf]

LAMBERT (E.). - Exploitation des forêts de Chêne-liège et des bois d'Olivier en Algérie. — Paris, 1860. $113 \mathrm{p}$.

LETREUCH-BELAROUCI (A.). - Caractérisation structurale des suberaies du Parc national de Tlemcen, régénération naturelle et gestion durable. - Université de Tlemcen, 2009. - 212 p. + annexes (Thèse Doctorat en Foresterie).

LETREUCH-BELAROUCI (A.). - Compréhension du processus de dégradation de la suberaie de Tlemcen et possibilités d'installation d'une réserve forestière. - Université de Tlemcen, 2002. - 205 p. (Thèse de Magistère).

LETREUCH-BELAROUCI (N.). - Les reboisements en Algérie et leur perspective d'avenir. - Alger : OPU, 1991. - Vol. I : 294 p. 
MAIRE (R.). - Carte phytogéographique de l'Algérie et de la Tunisie. - Notice, 1926. - 48 p. + 30 pl. + carte au $1 / 500000$.

MEDJAHDI (A.), LETREUCH-BELAROUCI (N.), BENABDELI (K.), MEDJAHDI (B.). - Gestion multifonctionnelle des formations forestières du Parc national de Tlemcen (Nord-Ouest Algérien). - Les Annales de l'INRGREF, numéro spécial, 12, 2008.

MESSAOUdEnE (M.). - Chêne zéen et Chêne afares. - Forêt algérienne, vol. II, 1996, pp. 18-25.

NATIVIDADE (J.V.). - Subériculture. - Nancy : Éditions de l’École nationale des Eaux et Forêts, 1956. $303 \mathrm{p}$.

NOUAL (M.S.). - Diagnostic et plan d'action pour une contribution de la forêt au développement intégré : étude du cas de la forêt de Mizrana (Algérie). - Montpellier : ENGREF, 1996. - 97 p. (Mastère spécialisé en sciences forestières, option forêts des régions chaudes).

PARC NATIONAL DE TLEMCEN. - La Suberaie de Hafir Tlemcen (Oranie Algérie). - Rapport du Parc national de Tlemcen, 2000. $-7 \mathrm{p}$.

SAUVAGE (Ch.). - Flore des suberaies marocaines (Catalogues des Cryptogames vasculaires et des phanérogames). - Travaux de l'Institut scientifique chérifien, Série botanique, n² 22, 1961.

TINTHOIN (R.). - Les aspects physiques du tell oranais : essai de morphologie de pays semi aride. - Oran : Ed. Fouquet, 1948. - 606 p.

VARELA (M.C.). - Analyse du rôle et du potentiel du Chêne-liège (Quercus suber) et de ses produits dans la région méditerranéenne, et examen d'une proposition visant à mettre au point une marque commerciale pour identifier les produits tirés du liège. - Portugal, 1997. - $4 \mathrm{p}$.

WARGE (L.), DUFRENE (M.). - Les réserves forestières. - OFFH. Circulaire du 23 juillet 1979 sur les Réserves forestières, 1996.

WWF. FONDS MONDIAL POUR LA NATURE. - Programme Méditerranée pour la sauvegarde des suberaies. WWF, 2004. - Plaquette, 2 p.

YESSAD (S.D.). - Le Chêne-liège et le liège dans les pays de la Méditerranée occidentale. - Louvain-laNeuve : ASBL Forêt wallonne, 2000. - 192 p.

ZERAÏA (L.). - Le Chêne-liège : phytosociologie, édaphologie, régénération et productivité. - Alger : INRF, 1982. - 159 p.

ZERAÏA (L.). - Essais d'interprétation comparative des données écologiques, phénologiques et de production subéro-ligneuse dans les forêts de Chêne-liège de provenance cristalline (France méridionale et Algérie). - Université Aix-Marseille, 1981. - 367 p. (Thèse Doctorat).

\section{Sthatégie de dÉueloppement et Consenuation des SUberhies des monts de tLemcen [hlgếrie] [hésumé]}

La suberaie est certainement reconnue pour ses caractéristiques intrinsèques et pour son aire de répartition de la Méditerranée occidentale et des côtes atlantiques comme un espace forestier intéressant à l'échelle européenne. L'intérêt biologique du Chêne-liège n'est plus à démontrer mondialement ; il est ainsi présent dans trois zones de la biodiversité sur dix identifiées pour le bassin de la Méditerranée. L’étude effectuée porte sur l'écologie et la dynamique d'un massif à caractère naturel, le massif de Hafir-Zariffet. Malheureusement l'intensification de la pression humaine, à travers le surpâturage et les incendies répétés, la combinaison de politiques inadaptées, de mauvaises pratiques de gestion des forêts et les évolutions du marché du liège ont entraîné la dégradation de cet habitat unique. Le travail a pour but de partager les connaissances sur la régénération des suberaies dégradées afin d'améliorer les conditions nécessaires à l'équilibre écologique, social et économique de ces écosystèmes et la remise en production. Un autre objectif poursuivi est aussi de démontrer et de discuter de la façon de raisonner la gestion durable des forêts de Chêne-liège, entraînant ainsi l'augmentation de leur valeur sociale et économique tout en abordant les menaces et risques de dégradation et de surexploitation. Gérer et restaurer les richesses naturelles des suberaies pour leur sauvegarde dans l'avenir est un enjeu essentiel. 


\section{A DEUELOPMENT AND CONSERUATION STRATEGY FOR THE CORK OAK FORESTS IN THE TLEMCEN HILLS [ALgERIR]} [Abstract]

Cork oak forests are recognized as a valuable forest space on the European scale. Cork oak is of biological interest worldwide; it is present in three of the ten biodiversity zones identified in the Mediterranean basin. Our study focuses on the ecology and the dynamics of a natural forest massif named "Hafir-Zariffet". Unfortunately, intensification of human activity, through overgrazing and repeated fires, the combination of inappropriate policies, bad forest management and cork market trends have led to the degradation of this unique habitat. The purpose of this work is to share knowledge on the regeneration of degraded cork oak forests in order to improve the conditions necessary to achieve ecological, social and economic balance for these ecosystems. We also show and discuss how sustainable management of the cork oak forests can be conceived so as to increase their social and economic value, and examine the threats and risks of degradation and overexploitation. Proper management and restoration of the cork oak forest resource are essential to their future conservation. 